OPEN ACCESS

Edited by:

Brock Aaron Arivett,

Middle Tennessee State University,

United States

Reviewed by:

Rafael Franco-Cendejas, National Institute of Rehabilitation Luis Guillermo Ibarra Ibarra, Mexico Steven Fiester,

University of South Carolina, United States

${ }^{*}$ Correspondence:

Charles Van der Henst charles.vanderhenst@vub.vib.be

Specialty section:

This article was submitted to Bacteria and Host, a section of the journal Frontiers in Cellular and

Infection Microbiology

Received: 05 October 2021 Accepted: 15 December 2021

Published: 24 January 2022

Citation:

Breine A, Van Gysel M, Elsocht M, Whiteway C, Philippe C, Quinet T, Valcek A, Wouters J, Ballet S and Van der Henst $C$ (2022) Antimicrobial Activity of a Repurposed

Harmine-Derived Compound on Carbapenem-Resistant Acinetobacter baumannii Clinical Isolates. Front. Cell. Infect. Microbiol. 11:789672. doi: 10.3389/fcimb.2021.789672

\section{Antimicrobial Activity of a Repurposed Harmine-Derived Compound on Carbapenem- Resistant Acinetobacter baumannii Clinical Isolates}

\begin{abstract}
Anke Breine ${ }^{1,2}$, Mégane Van Gysel ${ }^{3}$, Mathias Elsocht ${ }^{4}$, Clémence Whiteway ${ }^{1,2}$, Chantal Philippe ${ }^{5}$, Théo Quinet ${ }^{6,7}$, Adam Valcek ${ }^{1,2}$, Johan Wouters ${ }^{3}$, Steven Ballet ${ }^{4}$ and Charles Van der Henst ${ }^{1,2 *}$

${ }^{1}$ Microbial Resistance and Drug Discovery, Vlaams Instituut voor Biotechnologie-Vrije Universiteit Brussel (VIB-VUB) Center for Structural Biology, Vlaams Instituut voor Biotechnologie (VIB), Flanders Institute for Biotechnology, Brussels, Belgium, 2 Structural Biology Brussels, Vrije Universiteit Brussel (VUB), Brussels, Belgium, ${ }^{3}$ Namur Medicine and Drug Innovation Center (NAMEDIC), University of Namur (UNamur), Namur, Belgium, ${ }^{4}$ Research Group of Organic Chemistry, Departments of Chemistry and Bioengineering Sciences, Vrije Universiteit Brussel, Brussels, Belgium, ${ }^{5}$ Research Unit in the Biology of Microorganisms (URBM), NARILIS, University of Namur (UNamur), Namur, Belgium, ${ }^{6}$ Laboratory of Evolutionary Genetics and Ecology, URBE, University of Namur (UNamur), Namur, Belgium, ${ }^{7}$ Molecular Biology and Evolution, Universite' Libre de Bruxelles (ULB), Brussels, Belgium
\end{abstract}

Objectives: The spread of antibiotic resistant bacteria is an important threat for human health. Acinetobacter baumannii bacteria impose such a major issue, as multidrug- to pandrug-resistant strains have been isolated, rendering some infections untreatable. In this context, carbapenem-resistant $A$. baumannii bacteria were ranked as top priority by both $\mathrm{WHO}$ and CDC. In addition, A. baumannii bacteria survive in harsh environments, being capable of resisting to disinfectants and to persist prolonged periods of desiccation. Due to the high degree of variability found in A. baumannii isolates, the search for new antibacterials is very challenging because of the requirement of drug target conservation amongst the different strains. Here, we screened a chemical library to identify compounds active against several reference strains and carbapenem-resistant $A$. baumannii bacteria.

Methods: A repurposing drug screen was undertaken to identify A. baumannii growth inhibitors. One hit was further characterized by determining the $\mathrm{IC}_{50}$ and testing the activity on 43 modern clinical $A$. baumannii isolates, amongst which 40 are carbapenem-resistant.

Results: The repurposing screen led to the identification of a harmine-derived compound, called HDC1, which proves to have bactericidal activity on the multidrug-resistant AB5075-VUB reference strain with an $\mathrm{IC}_{50}$ of $48.23 \mu \mathrm{M}$. In addition, HDC1 impairs growth of 43 clinical $A$. baumannii isolates.

Conclusions: We identified a compound with inhibitory activity on all tested strains, including carbapenem-resistant clinical $A$. baumannii isolates.

Keywords: Acinetobacter baumannii, carbapenem-resistant, Gram-negative, pathogenic bacteria, repurposed compound, drug screening 


\section{INTRODUCTION}

The rise of antibiotic resistant bacteria is a global threat for healthcare, making it possible to succumb to diseases that were previously treatable (Wong et al., 2017). This has been acknowledged by both the World Health Organization (WHO) and the Centers for Disease Control and Prevention (CDC), which generated a list of drug-resistant pathogens for which new antibiotics are urgently needed (World Health Organization, 2017; CDC, 2019). The top priorities of these lists are antibiotic-resistant Acinetobacter baumannii bacteria (Whiteway et al., 2021).

A. baumannii is a Gram-negative, opportunistic bacterium, belonging to the ESKAPE group (Enterococcus faecium, Staphylococcus aureus, Klebsiella pneumoniae, Acinetobacter baumannii, Pseudomonas aeruginosa and Enterobacter spp.) of the most problematic nosocomial pathogens (Rice, 2008; Santajit and Indrawattana, 2016; Whiteway et al., 2021). While A. baumannii is a ubiquist (i.e. it can be found in soil, on human skin and in water sources), its presence especially imposes a threat in clinical settings (Vallenet et al., 2008; Wieland et al., 2018). This is due to a remarkable combination of resistance capabilities of A. baumannii, which is able to persist prolonged periods of desiccation, resist to disinfectants and to acquire drug resistance at a high rate (Da Silva and Domingues, 2016; Zeidler and Müller, 2019). Infections caused by A. baumannii commonly occur in immunocompromised patients and manifest as ventilator-assisted pneumonia, bacteremia and to a lesser extent skin or urinary tract infections (Weiner et al., 2016; Whiteway et al., 2021). Treatment of these infections becomes increasingly difficult, as multidrug-resistant, extensively drugresistant or even pandrug-resistant strains have been reported, with the latter being resistant to all available antibiotics, including carbapenems (Magiorakos et al., 2012; Gallagher et al., 2015). An important hurdle in the development of new antimicrobials against $A$. baumannii is the high diversity found between isolates, leading to a still open pan-genome (Adams et al., 2008).

In this context, the discovery of new inhibitory molecules, active against multidrug-resistant $A$. baumannii strains, is therefore crucial (World Health Organization, 2017; CDC, 2019). Harmine and harmine derivatives are $\beta$-carbolines that present pharmacological effects including anti-inflammatory, neuroprotective, antidiabetic, and antitumor activities (Zhang et al., 2020). $\beta$-carbolines also show anti-infective potential (Faheem et al., 2021). For natural and synthetic $\beta$-carboline analogs, antibacterial, antifungal, antiviral, antimalarial, antileishmanial and antitrypanosomal properties have been reported. Most studies deal with biological evaluation of the molecules and, only in some rare cases, potential mechanisms of action have been proposed. Mechanistic studies demonstrated that harmine effectively repressed the HSV-2 induced upregulation of Interleukin-1 $\beta$ (IL-1 $\beta$ ), Tumor Necrosis Factor$\alpha$ (TNF- $\alpha$ ), Interleukin-6 (IL-6), and Interleukin-8 (IL-8) (Chen et al., 2015). Mechanistically, substituted $\beta$-carbolines exhibit their anti-infective properties via several targets, including Topoisomerase II, DNA gyrase, MAPK, PfHsp90 or trypanothione reductase (Faheem et al., 2021).

For example, it has been shown that harmine exerts antileishmanial activity both in vitro and in vivo and causes necrosis by a nonspecific membrane damage in Leishmania donovani promastigotes (Lala et al., 2004). Harmine inhibits $P$. falciparum heat shock protein 90 by specific competition with its ATP-binding domain (Shahinas et al., 2012). Harmine analogs were further studied as binders to $P$. falciparum heat shock protein 90 ( $\mathrm{PfHsp90)}$ and two compounds inhibited the parasite in vitro at micromolar concentrations, reducing parasitemia, and prolonging the survival of $P$. berghei-infected mice (Bayih et al., 2016). Adequate substitution of the $\beta$-carboline scaffold led to enhanced nematicidal effect (Xia et al., 2019). Manzamine alkaloids, which are complex natural compounds consisting of a $\beta$-carboline nucleus, have been isolated and found to exhibit potent anti-infective activities by inhibiting target kinases like GSK-3 $\beta$ and MtSK (Ashoka et al., 2021).

9-substituted harmine derivatives show an inhibitory effect on dengue virus and impair the maturation and release of virus particles to the extracellular medium affecting the spreading of the infection (Quintana et al., 2016). Their mode of action still needs to be clarified. $9 \mathrm{~N}$-methylharmine neither affects viral adsorption-internalization events nor viral RNA synthesis. The antiviral activity is not related to the ability of the compound to downregulate p38 MAPK phosphorylation.

Harmine derivatives with 7,9- or 2,7,9-substituted 7-oxy-1methyl- $\beta$-carboline scaffolds inhibit activity of SerB2, an essential metabolic enzyme and suspected virulence factor of Mycobacterium tuberculosis (Frédérick et al., 2012; Carvalho et al., 2017; Carvalho et al., 2020; Pierson et al., 2020).

In this paper, we aimed at the discovery of a compound active against most clinical isolates. We performed a repurposing screen on a compound library, which led to the identification of a harmine-derived compound, called HDC1, with inhibitory activity on the growth of all the tested clinical isolates, amongst which 40 are carbapenem-resistant.

\section{MATERIAL AND METHODS}

\section{Compound Library and Synthesis of HDC1}

A compound library of the Namur Medicine \& Drug Innovation Center (NAMEDIC) was provided for a growth inhibition screen against A. baumannii. All compounds were dissolved in 100\% DMSO and used for the initial screen at $100 \mu \mathrm{M}$. The active compound HDC1 (1-methyl-2-benzyl-7-benzyloxy-9-benzyl- $\beta$ carbolin-2-ium bromide) was synthesized as previously described (Frédérick et al., 2012) with the following optimizations: 1 -methyl-7-hydroxy- $\beta$-carboline was synthesized by adding 1 -methyl-7-methoxy- $\beta$-carboline $(0.600$ g, 2.83 mmol, 1 equiv.), hydrobromic acid $\left(12 \mathrm{ml}, 48 \%\right.$ in $\mathrm{H}_{2} \mathrm{O}$ ) and acetic acid ( $12 \mathrm{ml})$ into a round-bottom flask, equipped with reflux condenser. The mixture was refluxed overnight under argon atmosphere and subsequently added to distilled water $(100 \mathrm{ml})$. The precipitate was isolated via filtration, washed with 
cold water and dried under vacuum yielding 1-methyl-7hydroxy- $\beta$-carboline with $81 \%(0.454 \mathrm{~g})$ yield. ${ }^{1} \mathrm{H}-\mathrm{NMR}(500$ MHz, DMSO-d6) $\delta$ (ppm): 12.59 (s, 1H), 10.62 (s, 1H), 8.39-8.29 (m, 2H), $8.21(\mathrm{~d}, \mathrm{~J}=8.7 \mathrm{~Hz}, 1 \mathrm{H}), 7.03(\mathrm{~d}, \mathrm{~J}=1.7 \mathrm{~Hz}, 1 \mathrm{H}), 6.89$ (dd, J = 8.7, 1.7 Hz, 1H), 2.94 (s, 3H). Next, 1-methyl-2-benzyl-7benzyloxy-9-benzyl- $\beta$-carbolin-2-ium bromide was synthesized. First 1-methyl-7-hydroxy- $\beta$-carboline $(0.500 \mathrm{~g}, 2.52 \mathrm{mmol}, 1$ equiv.) was dissolved in anhydrous $\mathrm{N}, \mathrm{N}$,-dimethylformamide (20 $\mathrm{ml}$ ) into a flame-dried microwave vial under argon atmosphere. Then KOtBu (0.849 g, $7.57 \mathrm{mmol}, 3$ equiv.) was added and the mixture was stirred for 30 minutes at room temperature. Subsequently benzyl bromide $(3.00 \mathrm{ml}, 25.2 \mathrm{mmol}, 10$ equiv.) was added and the mixture was heated overnight at $75^{\circ} \mathrm{C}$. Afterwards the crude mixture was filtered, and the precipitate was washed with $\mathrm{CH}_{2} \mathrm{Cl}_{2}$. The volatiles in the filtrate were removed under reduced pressure and the crude product was subjected to column chromatography (cyclohexane/ethyl acetate) yielding the desired product with $46 \%$ (0.638 g) yield. ${ }^{1} \mathrm{H}-\mathrm{NMR}$ (500 MHz, DMSO-d6) $\delta$ (ppm): $8.86(\mathrm{~d}, \mathrm{~J}=6.6 \mathrm{~Hz}$, $1 \mathrm{H}), 8.72(\mathrm{~d}, \mathrm{~J}=6.6 \mathrm{~Hz}, 1 \mathrm{H}), 8.49(\mathrm{~d}, \mathrm{~J}=8.8 \mathrm{~Hz}, 1 \mathrm{H}), 7.57$ (d, J = $2.1 \mathrm{~Hz}, 1 \mathrm{H}), 7.50-7.46(\mathrm{~m}, 2 \mathrm{H}), 7.40-7.25(\mathrm{~m}, 9 \mathrm{H}), 7.23$ (dd, J = 8.8, 2.1 Hz, 1H), 7.12 (d, J = 7.2 Hz, 2H), 6.99 (d, J = 7.2 Hz, 2H), $6.02(\mathrm{~s}, 2 \mathrm{H}), 5.99$ (s, 2H), 5.27 (s, 2H), 2.85 (s, 3H). ${ }^{13} \mathrm{C}-\mathrm{NMR}$ (126 MHz, DMSO-d6) $\delta$ (ppm): 162.8 (Cq), 148.0 (Cq), 139.6 $(\mathrm{Cq}), 137.5(\mathrm{Cq}), 136.2(\mathrm{Cq}), 135.4(\mathrm{Cq}), 134.7(\mathrm{Cq}), 133.5(\mathrm{Cq})$, $129.1(\mathrm{CH}), 129.0(\mathrm{CH}), 128.5(\mathrm{CH}), 128.3(\mathrm{CH}), 127.5(\mathrm{CH})$, $126.6(\mathrm{CH}), 125.4(\mathrm{CH}), 124.9(\mathrm{CH}), 114.7(\mathrm{CH}), 113.7(\mathrm{CH})$, $112.8(\mathrm{Cq}), 95.0(\mathrm{CH}), 70.1(\mathrm{CH} 2), 59.8(\mathrm{CH} 2), 48.3(\mathrm{CH} 2), 16.0$ (CH3). The spectroscopic data were in accordance with those reported by Frédérick et al. (2012).

\section{Strains, Media and OD $_{600 n m}$ Measurements}

Taking into account the high genomic dynamics observed for $A$. baumannii bacteria, we used the proposed modern nomenclature in the field (Gallagher et al., 2015) by renaming the sub-cultured isolates by adding "-VUB" to the strain name, although these strains are a priori identical or very similar to the ones provided by the National Reference Center (NRC)
Laboratory for Antibiotic-Resistant Gram-Negative Bacilli (CHU UCL Namur, Yvoir, Belgium). All A. baumannii reference strains and clinical isolates were cultured at $37^{\circ} \mathrm{C}$ in LB broth media under agitation $(355 \mathrm{cpm})$ conditions for $16 \mathrm{~h}$ before subsequent experiments. For $\mathrm{IC}_{50}$ determination, bacterial cells corresponding to $\mathrm{OD}_{600 \mathrm{~nm}}=0.1$ were transferred to a 96 well flat bottom plate (Greiner, Austria) containing varying concentrations of HDC1 $(0.1 ; 1 ; 10 ; 25 ; 50 ; 75 ; 100$ and 1000 $\mu \mathrm{M})$ in LB broth media with $1 \%$ DMSO. For determination of the activity of HDC1 on the 43 clinical A. baumannii isolates, bacterial cells corresponding to $\mathrm{OD}_{600 \mathrm{~nm}}=0.1$ were transferred to a 96 well flat bottom plate (Greiner, Austria) containing 100 $\mu \mathrm{M}$ of HDC1 in LB broth media with $1 \%$ DMSO. For both experiments, the positive control included bacterial cells corresponding to $\mathrm{OD}_{600 \mathrm{~nm}}=0.1$ of the used strains in $\mathrm{LB}$ broth media with $1 \%$ DMSO. The negative control included both LB broth media and LB broth media supplemented with 1\% DMSO. Collection of data was done using the Cytation 1 (BioTek, United States). The $\mathrm{OD}_{600 \mathrm{~nm}}$ absorbance of all bacterial cultures was measured every $30 \mathrm{~min}$ for $24 \mathrm{~h}$ at a temperature of $37^{\circ} \mathrm{C}$ and agitation of $355 \mathrm{cpm}$ (cycles per minute). In all analysis, growth inhibition is defined as decreasing absorbance values over time compared to the positive control. The classification of strains into inhibition levels is based on the ratio of the final absorbance value of the HDC1-treated bacteria $\left(\mathrm{OD}_{600 \mathrm{~nm} ; \mathrm{HDCl}}\right)$ compared to the final absorbance value of the non-treated control $\left(\mathrm{OD}_{600 \mathrm{~nm}}\right.$; control $)$ as followed: (i) low inhibition $\left(\mathrm{OD}_{600 \mathrm{~nm} ;} \mathrm{HDC} 1>50 \%\right.$ of $\mathrm{OD}_{600 \mathrm{~nm}}$; control); (ii) intermediate inhibition $\left(10 \%\right.$ of $\mathrm{OD}_{600 \mathrm{~nm}}$; control $<\mathrm{OD}_{600 \mathrm{~nm} \text {; } \mathrm{HDCl}}<$ or $=50 \%$ of $\mathrm{OD}_{600 \mathrm{~nm}}$; control $)$ and (iii) complete inhibition $\left(\mathrm{OD}_{600 \mathrm{~nm}}\right.$, $\mathrm{HDC} 1<$ or $=10 \% \mathrm{OD}_{600 \mathrm{~nm}}$; control). The data were measured in biological triplicate.

\section{CFU Determination}

After absorbance measurements for $\mathrm{IC}_{50}$ determination, the positive control (AB5075-VUB grown in LB with 1\% DMSO) and AB5075-VUB grown in presence of $100 \mu \mathrm{M}$ HDC1, were resuspended in $\mathrm{PBS}$, brought to the same $\mathrm{OD}_{600 \mathrm{~nm}}$ and plated on LB agar plates in appropriate dilutions. After $16 \mathrm{~h}$ incubation of<smiles>COc1ccc2c(c1)[nH]c1c(C)nccc12</smiles>

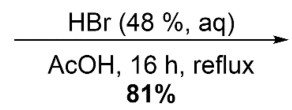<smiles>Cc1nccc2c1[nH]c1cc(O)ccc12</smiles>

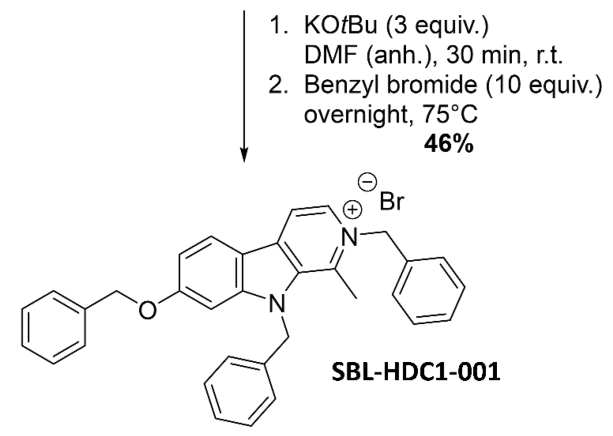


the $\mathrm{LB}$ agar plates at $37^{\circ} \mathrm{C}$, CFUs were counted to assess any bacteriostatic or bactericidal effect. All data was measured in biological triplicate. To estimate the initial bacterial load, the relationship between the absorbance at $\mathrm{OD}_{600 \mathrm{~nm}}$ and CFUs was determined for the strain AB5075-VUB. This was done by plating serial dilutions of bacterial cultures with a known $\mathrm{OD}_{600 \mathrm{~nm}}$ on $\mathrm{LB}$ agar and counting the corresponding CFUs (see Supplementary Table 1).

\section{$\mathrm{IC}_{50}$ Calculation}

For the determination of the $\mathrm{IC}_{50}$, GraphPad Prism 9 (GraphPad Software, LLC) was used. After 20 hours, the $\mathrm{OD}_{600 \mathrm{~nm}}$ absorbance kinetic data were obtained and normalized using the positive control absorbance value as $100 \%$ viability and the absorbance value of $1 \mathrm{mM} \mathrm{HDC1}$ as $0 \%$ viability. The analysis was then done on the normalized data by nonlinear regression curve fitting. The $\mathrm{IC}_{50}$ value is shown with a $95 \%$ confidence interval (CI).

\section{Statistical Analysis}

All data shown are represented as mean \pm standard deviation of three biological replicates, except otherwise stated. CFU were statistically analyzed by an unpaired t-test. All growth curves were statistically analyzed by a Mann Whitney test. The $\mathrm{p}$ values $<0.05$ were considered significant.

\section{RESULTS}

\section{Repurposing Screen Reveals Compound With Inhibitory Activity on AB5075-VUB}

The initial screen from a chemical library of the Namur Medicine \& Drug Innovation Center from the University of Namur (UNamur) aimed at the identification of growth inhibitors for problematic multidrug-resistant $A$. baumannii strains. The strain initially used for this screen is a multidrug-resistant $A$. baumannii reference strain, AB5075-VUB. This strain is a derivative from the parental strain AB5075 that was clonally isolated in our laboratory at the VUB (Vrije Universiteit Brussel).

The screen showed complete growth inhibition by one compound called HDC1, for it is a harmine-derived compound (Figure 1). HDC1 was originally synthesized for an anticancer drug screen. Interestingly, compounds with anticancer activity have lately been explored as potential antimicrobials (Cheng et al., 2019). In line with this tendency, HDC1 was further characterized for other activities.
A<smiles>Cc1c2c(cc[n+]1[B-]c1ccccc1)c1ccc(OCc3ccccc3)cc1n2Cc1ccccc1</smiles>

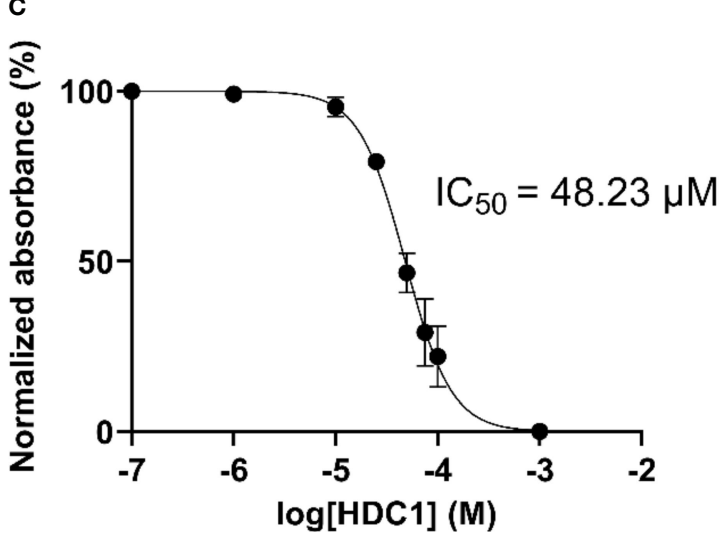

B
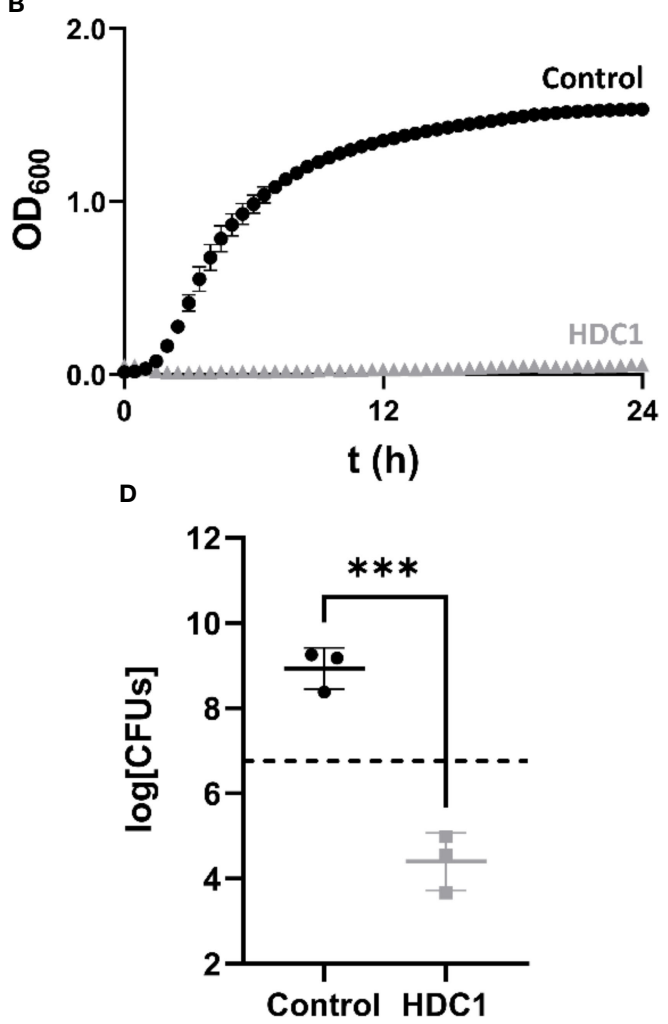

FIGURE 1 | (A) Structure of HDC1. (B) Growth curve of AB5075-VUB in presence (grey triangles) and absence (black spheres) of $100 \mu \mathrm{M}$ of HDC1. (C) Non-linear regression curve of normalized absorbance reads in function of compound concentration. (D) Number of viable bacteria after $24 \mathrm{~h}$ incubation without and with 100 $\mu \mathrm{M}$ of HDC1. The initial bacterial load is represented by the dashed line on the plot. All data points in this figure are shown as mean \pm standard deviation of three independent biological replicates. ${ }^{\star \star \star} \mathrm{p}<0.001$. 


\section{HDC1 Has Bactericidal Activity on AB5075-VUB}

To determine the potency of the compound, the minimum concentration required for $50 \%$ growth inhibition, $\mathrm{IC}_{50}$, was determined for the AB5075-VUB strain. The analysis showed that $\mathrm{HDC} 1$ has an $\mathrm{IC}_{50}$ of $48.23 \mu \mathrm{M}$ (95\% CI 44.76-51.83) (Figure 1C). To determine the potential antimicrobial effect of HDC1 on AB5075-VUB viability, the strain was grown in presence of $100 \mu \mathrm{M}$ of HDC1. After $24 \mathrm{~h}$ incubation, the bacteria were resuspended in fresh media without the compound and plated on LB agar plates for CFUs enumeration. After $24 \mathrm{~h}$ incubation, the number of recovered bacteria is significantly different when bacteria are incubated with the compound compared to the control group (Figure 1D). While a 40 -fold increase in CFUs is observed for the control group, a 1000-fold decrease of CFUs is observed in the presence of HDC1, compared to the initial bacterial inoculum. This shows a significant bactericidal activity of HDC1 on the multidrugresistant AB5075-VUB reference strain.

\section{HDC1 Has Broad Inhibitory Activity on All the Tested Clinical Isolates}

A. baumannii has a highly dynamic genome (Wright et al., 2016). The presence of mobile genetic elements and the efficient acquisition of genes through horizontal gene transfer are not only responsible for the pathogen's success in obtaining drug resistance and environmental persistence, but they are also the reason isolates have become more and more diverse (Adams et al., 2008; Imperi et al., 2011; Wright et al., 2016). The core genome of the pathogen's strains is reported to be relatively small and the accessory genome of strains can be up to $25-46 \%$ unique (Adams et al., 2008; Imperi et al., 2011). This heterogeneity found in isolates renders the search for antimicrobial compounds increasingly difficult. It is therefore important for a new potential antimicrobial to exert its activity not only on a few $A$. baumannii strains, but on a multitude of diverse and clinically relevant isolates.

To determine the activity of HDC1 on recent isolates, we used 43 recent A. baumannii clinical isolates, amongst which 40 are carbapenem-resistant strains (Valcek et al., 2021). In addition to these recent clinical strains, clonal isolates of three frequently used reference strains were also included. Two of these strains, ATCC19606 and ATCC17978, are older type strains, compared to the more recent and multidrug-resistant AB5075 reference strain (Gallagher et al., 2015; Harding et al., 2018). The third reference strain, DSM30011-VUB, is an environmental isolate (Repizo et al., 2017).

The growth of all tested strains was impaired by the presence of HDC1 (Figure 2), with low, intermediate, or complete inhibition levels. An overview of the different resistance profiles against HDC1 can be found in Table 1. Complete inhibition of growth is observed for all three reference strains and 13 recent isolates, while most of the clinical isolates show an intermediate inhibition profile. The AB193-VUB isolate showed the least sensitivity to HDC1. No correlation could be established between the sensitivity of the tested strains to the HDC1 compound, and the presence of the antibiotic-resistance genes found in the modern clinical isolates tested.

\section{DISCUSSION}

In this study, a repurposing drug screen led to the discovery of a compound with inhibitory activity on the growth of a multidrugresistant Acinetobacter baumannii reference strain, AB5075-VUB. This compound, named HDC1, is a harmine-derivative previously designed to have anticancer properties. The anticancer screen showed that the compound acts as a protein synthesis inhibitor at a concentration of $0.7 \mu \mathrm{M}$ (Frédérick et al., 2012). In our study, HDC1 was shown to have bactericidal activity on AB5075-VUB with an $\mathrm{IC}_{50}$ of $48.23 \mu \mathrm{M}$. As the compound was previously found to be cytotoxic in HEPG2 cells at $50 \mu \mathrm{M}$, this limits the potential of HDC1 as a new antimicrobial without further modification of the molecule (Marx et al., 2019). Differences in active concentrations of HDC1 between cancer and bacterial cells could be attributed to cell size and target(s) presence and/or abundance, altered metabolism states and the presence or absence of posttranslational modifications. However, as multidrug-, extensively drug- and pandrug-resistant $A$. baumannii strains are emerging and spreading, every option deems to be explored.

Due to the high diversity between $A$. baumannii isolates, one of the main hurdles in the discovery of new compounds is to find compounds capable of targeting the majority of the isolates. Here, we report a compound to have inhibitory activity on all the tested and recent carbapenem-resistant A. baumannii isolates. Our test shows various degrees of growth inhibition: from complete to intermediate to only slight inhibition. In addition, the 4 reference strains used in our study all show a high degree of sensitivity to HDC1. Taken together, this raises the following questions (i) what contributes to this difference in growth inhibition levels, (ii) what could be the target(s) of HDC1 and (iii) why is the whole AB5075-VUB bacterial population not killed by $\mathrm{HDC} 1$ in the tested conditions, since a significant bactericidal effect is observed? The phase variation observed in AB5075 might be the answer to the last question. Phase variation is a morphological feature of $A$. baumannii colonies where 2 different opacity phenotypes are observed: opaque and translucent (Tipton et al., 2015). Opaque and translucent colonies exhibit multiple phenotypic differences, including cell morphology, surface motility, biofilm formation and virulence, but of particular interest also a difference in resistance to antibiotics and thus potentially to HDC1 (Tipton et al., 2015; Chin et al., 2018). Interestingly, a recent study showed that HDC1 inhibits the phosphoserine phosphatase of Mycobacterium tuberculosis, MtSerB2 (Pierson et al., 2020). MtSerB2 catalyzes the last step in the L-serine biosynthetic pathway and is involved in immune evasion mechanisms of $M$. tuberculosis (Pierson et al., 2020). In AB5075-VUB, a homolog of MtSerB2 is present: AbSerB. This indicates a putative target of HDC1 in A. baumannii. A possible explanation for the different growth inhibition profiles of the clinical isolates could be the presence of mutations in the $\operatorname{ser} B$ gene. Although $\operatorname{ser} B$ is highly 
A

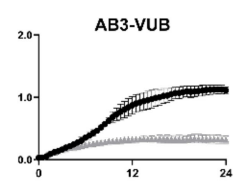

AB21-VuB
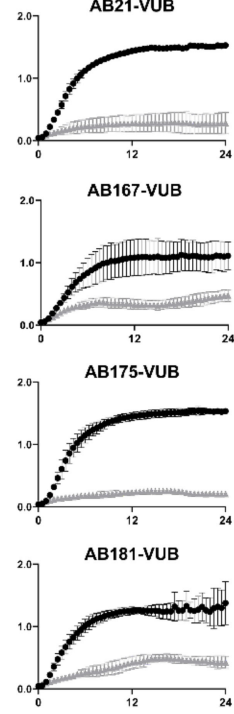

AB189-VUB

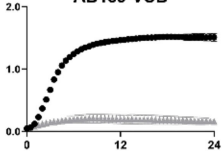

AB214-VUB

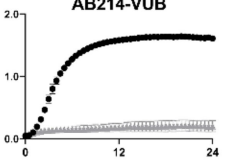

AB222-VUB
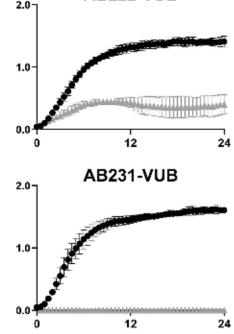

B

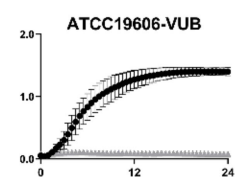

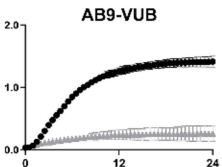

AB32-vuB
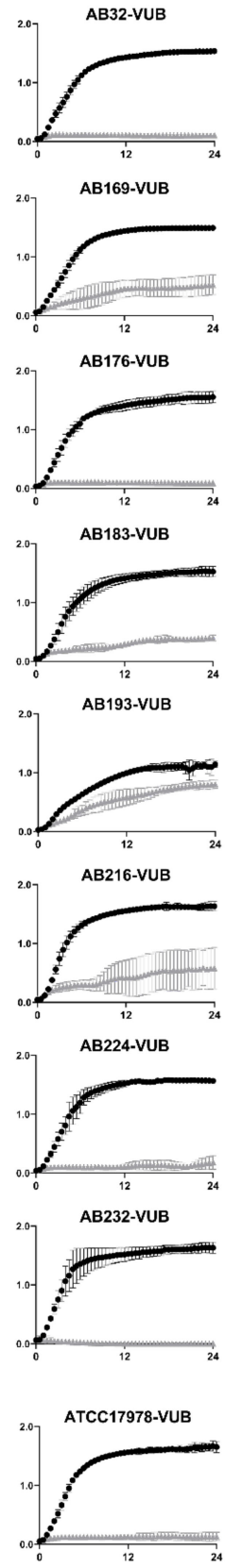

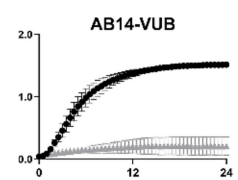

AB36-VUB

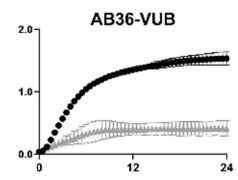

AB171-VUB
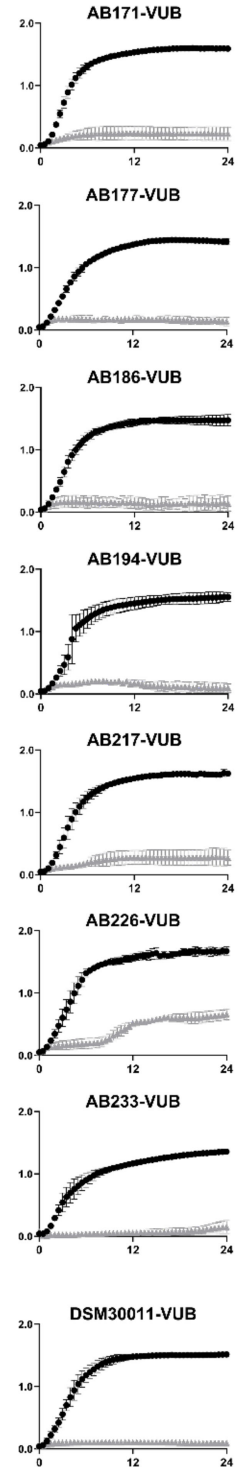
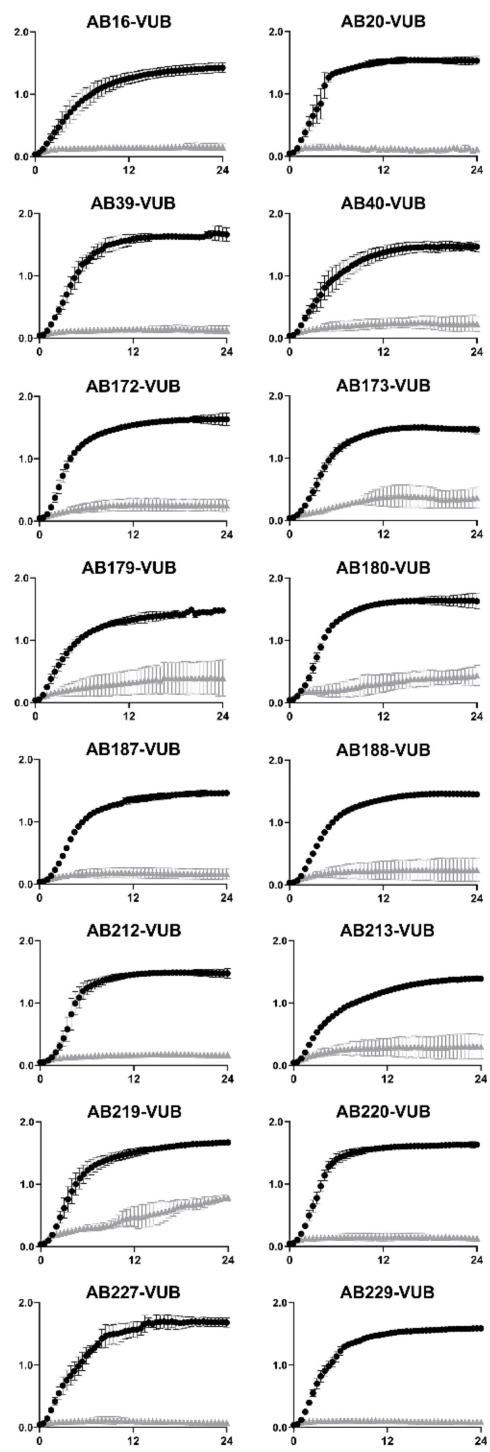

FIGURE 2 | (A) Growth curves of 43 clinical A. baumannii isolates. (B) Growth curves of three A. baumannii reference strains (ATCC19606-VUB, ATCC17978-VUB and DSM30011-VUB). All strains were incubated with $100 \mu \mathrm{M}$ of HDC1 (grey triangles) or without HDC1 (black spheres) for $24 \mathrm{~h}$. All data points are shown as mean \pm standard deviation of three independent biological replicates. X axis and y axis correspond to respectively time $(\mathrm{h})$ and absorbance values measured at $600 \mathrm{~nm}$.

conserved in all our tested A. baumannii strains (sequence identity between $98.45-100 \%$ ), no correlation could be found between mutations in $\operatorname{ser} B$ and the growth inhibition profiles of the A. baumannii isolates (Supplementary Table 2). The target(s) of HDC1 in A. baumannii remain(s) to be determined. Interestingly, it has been shown for M. tuberculosis that certain 
TABLE 1 | Growth inhibition levels on three reference strains and 43 clinical isolates of $A$. baumannii.

\begin{tabular}{|c|c|c|}
\hline \multicolumn{3}{|c|}{ Level of growth inhibition } \\
\hline Low & Intermediate & Complete \\
\hline AB193-VUB & $\begin{array}{l}\text { AB3-VUB } \\
\text { AB9-VUB } \\
\text { AB14-VUB } \\
\text { AB16-VUB } \\
\text { AB21-VUB } \\
\text { AB36-VUB } \\
\text { AB40-VUB } \\
\text { AB167-VUB } \\
\text { AB169-VUB } \\
\text { AB171-VUB } \\
\text { AB172-VUB } \\
\text { AB173-VUB } \\
\text { AB175-VUB } \\
\text { AB179-VUB } \\
\text { AB180-VUB } \\
\text { AB181-VUB } \\
\text { AB183-VUB } \\
\text { AB187-VUB } \\
\text { AB188-VUB } \\
\text { AB212-VUB } \\
\text { AB213-VUB } \\
\text { AB214-VUB } \\
\text { AB216-VUB } \\
\text { AB217-VUB } \\
\text { AB219-VUB } \\
\text { AB222-VUB } \\
\text { AB224-VUB } \\
\text { AB226-VUB } \\
\text { AB233-VUB }\end{array}$ & $\begin{array}{l}\text { ATCC19606-VUB } \\
\text { ATCC17978-VUB } \\
\text { DSM30011-VUB } \\
\text { AB20-VUB } \\
\text { AB32-VUB } \\
\text { AB39-VUB } \\
\text { AB176-VUB } \\
\text { AB177-VUB } \\
\text { AB186-VUB } \\
\text { AB189-VUB } \\
\text { AB194-VUB } \\
\text { AB220-VUB } \\
\text { AB227-VUB } \\
\text { AB229-VUB } \\
\text { AB231-VUB } \\
\text { AB232-VUB }\end{array}$ \\
\hline
\end{tabular}

The classification of strains into inhibition levels is based on the ratio of the final absorbance value of the HDC1-treated bacteria $\left(O D_{600 n m ; ~ H D C 1}\right)$ compared to the final absorbance value of the non-treated control $\left(O D_{600 n m ;}\right.$ control) as followed: (i) low inhibition $\left(O D_{600 n m ;} H D C 1>50 \%\right.$ of $O D_{600 n m \text {; control }}$ ); (ii) intermediate inhibition (10\% of $O D_{600 \mathrm{~nm} \text {; control }}<O D_{600 \mathrm{~nm}}$; $H D C 1<$ or $=50 \%$ of $O D_{600 \mathrm{~nm}}$; control) and (iii) complete

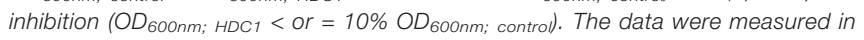
biological triplicate.

compounds are more efficient at inhibiting growth of the bacterium itself, than inhibiting the enzyme only, suggesting different mechanisms of action or intracellular accumulation of the compounds (Haufroid and Wouters, 2019). Additional resistance mechanisms, potentially countering such effects, could explain the higher resistance to HDC1 of some clinical isolates. Nevertheless, HDC1 has broad activity on all the tested recent clinical A. baumannii isolates of our study, which prompts the further exploration of this compound and/or cognate putative target in A. baumannii for drug discovery.

\section{CONCLUSION}

In conclusion, HDC1 is a potent harmine-derived compound with antibacterial activity identified using a multidrug-resistant A. baumannii strain, that also significantly inhibits the growth of diverse, recent, and carbapenem-resistant clinical $A$. baumannii isolates.

\section{DATA AVAILABILITY STATEMENT}

The original contributions presented in the study are included in the article/Supplementary Material. Further inquiries can be directed to the corresponding author.

\section{AUTHOR CONTRIBUTIONS}

Drafting of the manuscript, $\mathrm{AB}$ and $\mathrm{CV}$. Corrections of the manuscript, $\mathrm{AB}, \mathrm{MV}, \mathrm{ME}, \mathrm{CP}, \mathrm{JW}, \mathrm{SB}$, and $\mathrm{CV}$. HDC1 production, $\mathrm{ME}$ and SB. Preliminary drug screen, TQ, CP, and CV. Bioinformatics, $\mathrm{AB}$ and $\mathrm{CH}$. Experiments, $\mathrm{AB}, \mathrm{CW}$, and CV. Data analyses, $\mathrm{AB}, \mathrm{MV}$, JW, and CV. All authors contributed to the article and approved the submitted version.

\section{FUNDING}

$\mathrm{AB}$ is recipient of a $\mathrm{PhD}$ fellowship Strategic Basic Research of the Research Foundation - Flanders (FWO, File number: 77258). $\mathrm{ME}$ and $\mathrm{SB}$ acknowledge financial support of the Research Council at the Vrije Universiteit Brussel (VUB) through the IRP funding scheme. CV acknowledges the financial support from the Flanders Institute for Biotechnology (VIB). This project has received funding from the European Union's Horizon 2020 research and innovation program under the Marie SklodowskaCurie grant agreement No 748032 .

\section{ACKNOWLEDGMENTS}

We are grateful to Pr. Tom Coenye for providing us the strain AB5075 and Dr. Suzana Salcedo for the strains ATCC19606, ATCC17978 and DSM30011, as well as for fruitful discussions. We would like to thank Pr. Pierre Bogaerts, Pr. Te-din Huang and Pr. Olivier Denis, from the National Reference Center (NRC) Laboratory for Antibiotic-Resistant Gram-Negative Bacilli (CHU UCL Namur, Yvoir, Belgium) for providing the recent clinical isolates of $A$. baumannii. We also thank the members of Namedic (NARILIS-UNamur), in particular Pr. B. Masereel and L. Pochet for their research that allowed setting up the chemical library used in the present screening.

\section{SUPPLEMENTARY MATERIAL}

The Supplementary Material for this article can be found online at: https://www.frontiersin.org/articles/10.3389/fcimb.2021.789672/ full\#supplementary-material

Supplementary Table 1 | Relationship between the absorbance $\left(\mathrm{OD}_{600 \mathrm{~nm}}\right)$ and the colony forming units (CFUs) per $\mathrm{ml}$ for the strain AB5075-VUB. The values are shown for overnight cultures that were diluted for accurate absorbance measurements. Rep $=$ Biological replicate. At an $\mathrm{OD}_{600 \mathrm{~nm}}=1$, the $\mathrm{CFU} / \mathrm{ml}$ corresponds to $3.2 \pm 0.3$ $10^{8} \mathrm{CFU} / \mathrm{ml}$

Supplementary Table 2 | Percentage of nucleotide identity of the serB gene in 43 clinical isolates and four reference strains compared to the reference AB5075UW (NZ_CP008706.1) with AA substitution where applicable. 


\section{REFERENCES}

Adams, M. D., Goglin, K., Molyneaux, N., Hujer, K. M., Lavender, H., Jamison, J. J., et al. (2008). Comparative Genome Sequence Analysis of MultidrugResistant Acinetobacter Baumannii. J. Bacteriol. 190, 8053-8064. doi: 10.1128/JB.00834-08

Ashoka, P., Faheem,, Kumar, B., Chander, S., Chandra Sekhar, K., and Sankaranarayanan, M. (2021). Anti-Infective Potential of Manzamine Alkaloids - A Review. Med. Chem. doi: 10.2174/157340641766621 0803101740

Bayih, A. G., Folefoc, A., Mohon, A. N., Eagon, S., Anderson, M., and Pillai, D. R. (2016). In Vitro and In Vivo Anti-Malarial Activity of Novel Harmine-Analog Heat Shock Protein 90 Inhibitors: A Possible Partner for Artemisinin. Malar. J. 15, 1-11. doi: 10.1186/s12936-016-1625-7

Carvalho, A., Chu, J., Meinguet, C., Kiss, R., Vandenbussche, G., Masereel, B., et al. (2017). A Harmine-Derived Beta-Carboline Displays Anti-Cancer Effects In Vitro by Targeting Protein Synthesis. Eur. J. Pharmacol. 805, 25-35. doi: 10.1016/j.ejphar.2017.03.034

Carvalho, A., Viaene, J., Vandenbussche, G., De Braekeleer, K., Masereel, B., Wouters, J., et al. (2020). A New Potential Anti-Cancer Beta-Carboline Derivative Decreases the Expression Levels of Key Proteins Involved in Glioma Aggressiveness: A Proteomic Investigation. Drug Dev. Res. 81, 3242. doi: $10.1002 /$ ddr.21600

CDC. (2019). Antibiotic Resistance Threats in the United States. Atlanta, GA: U.S. Department of Health and Human Services, CDC. doi: 10.15620/cdc:82532

Cheng, Y. S., Sun, W., Xu, M., Shen, M., Khraiwesh, M., Sciotti, R. J., et al. (2019). Repurposing Screen Identifies Unconventional Drugs With Activity Against Multidrug Resistant Acinetobacter Baumannii. Front. Cell Infect. Microbiol. 9:438. doi: $10.3389 /$ fcimb.2018.00438

Chen, D., Su, A., Fu, Y., Wang, X., Lv, X., Xu, W., et al. (2015). Harmine Blocks Herpes Simplex Virus Infection Through Downregulating Cellular NF- $\kappa b$ and MAPK Pathways Induced by Oxidative Stress. Antiviral Res. 123, 27-38. doi: $10.1016 /$ j.antiviral.2015.09.003

Chin, C. Y., Tipton, K. A., Farokhyfar, M., Burd, E. M., Weiss, D. S., and Rather, P. N. (2018). A High-Frequency Phenotypic Switch Links Bacterial Virulence and Environmental Survival in Acinetobacter Baumannii. Nat. Microbiol. 3, 563569. doi: 10.1038/s41564-018-0151-5

Da Silva, G., and Domingues, S. (2016). Insights on the Horizontal Gene Transfer of Carbapenemase Determinants in the Opportunistic Pathogen Acinetobacter baumannii. Microorganisms 4:29. doi: 10.3390/microorganisms4030029

Faheem,, Kumar, B. K., Sekhar, K. V. G. C., Kunjiappan, S., Jamalis, J., BalañaFouce, R., et al. (2021). Recent Update on the Anti-Infective Potential of BCarboline Analogs. Mini Rev. Med. Chem. 21. doi: 10.2174/ 1389557520666201001130114

Frédérick, R., Bruyére, C., Vancraeynest, C., Reniers, J., Meinguet, C., Pochet, L., et al. (2012). Novel Trisubstituted Harmine Derivatives With Original In Vitro Anticancer Activity. J. Med. Chem. 55, 6489-6501. doi: 10.1021/jm300542e

Gallagher, L. A., Ramage, E., Weiss, E. J., Radey, M., Hayden, H. S., Held, K. G., et al. (2015). Resources for Genetic and Genomic Analysis of Emerging Pathogen Acinetobacter Baumannii. J. Bacteriol. 197, 2027-2035. doi: 10.1128/JB.00131-15

Harding, C. M., Hennon, S. W., and Feldman, M. F. (2018). Uncovering the Mechanisms of Acinetobacter Baumannii Virulence. Nat. Rev. Microbiol. 176, 139-148. doi: 10.1038/nrmicro.2017.148

Haufroid, M., and Wouters, J. (2019). Targeting the Serine Pathway: A Promising Approach Against Tuberculosis? Pharmaceuticals 12, 1-20. doi: 10.3390/ ph12020066

Imperi, F., Antunes, L. C. S., Blom, J., Villa, L., Iacono, M., Visca, P., et al. (2011). The Genomics of Acinetobacter Baumannii: Insights Into Genome Plasticity, Antimicrobial Resistance and Pathogenicity. IUBMB Life 63, 1068-1074. doi: 10.1002/iub.531

Lala, S., Pramanick, S., Mukhopadhyay, S., Bandyopadhyay, S., and Basu, M. K. (2004). Harmine: Evaluation of its Antileishmanial Properties in Various Vesicular Delivery Systems. J. Drug Target. 12, 165-175. doi: 10.1080/ 10611860410001712696

Magiorakos, A. P., Srinivasan, A., Carey, R. B., Carmeli, Y., Falagas, M. E., Giske, C. G., et al. (2012). Multidrug-Resistant, Extensively Drug-Resistant and Pandrug-Resistant Bacteria: An International Expert Proposal for Interim
Standard Definitions for Acquired Resistance. Clin. Microbiol. Infect. 18, 268-281. doi: 10.1111/j.1469-0691.2011.03570.x

Marx, S., Van Gysel, M., Breuer, A., Dal Maso, T., Michiels, C., Wouters, J., et al. (2019). Potentialization of Anticancer Agents by Identification of New Chemosensitizers Active Under Hypoxia. Biochem. Pharmacol. 162, 224236. doi: 10.1016/j.bcp.2019.01.004

Pierson, E., Haufroid, M., Gosain, T. P., Chopra, P., Singh, R., and Wouters, J. (2020). Identification and Repurposing of Trisubstituted Harmine Derivatives as Novel Inhibitors of Mycobacterium Tuberculosis Phosphoserine Phosphatase. Molecules 25, 1-17. doi: 10.3390/molecules25020415

Quintana, V. M., Piccini, L. E., Panozzo Zénere, J. D., Damonte, E. B., Ponce, M. A., and Castilla, V. (2016). Antiviral Activity of Natural and Synthetic $\beta$ Carbolines Against Dengue Virus. Antiviral Res. 134, 26-33. doi: 10.1016/ j.antiviral.2016.08.018

Repizo, G. D., Viale, A. M., Borges, V., Cameranesi, M. M., Taib, N., Espariz, M., et al. (2017). The Environmental Acinetobacter Baumannii Isolate DSM30011 Reveals Clues Into the Preantibiotic Era Genome Diversity, Virulence Potential, and Niche Range of a Predominant Nosocomial Pathogen. Genome Biol. Evol. 9, 2292-2307. doi: 10.1093/gbe/evx162

Rice, L. B. (2008). Federal Funding for the Study of Antimicrobial Resistance in Nosocomial Pathogens: No ESKAPE. J. Infect. Dis. 197, 1079-1081. doi: $10.1086 / 533452$

Santajit, S., and Indrawattana, N. (2016). Mechanisms of Antimicrobial Resistance in ESKAPE Pathogens. BioMed. Res. Int. 2016. doi: 10.1155/2016/2475067

Shahinas, D., MacMullin, G., Benedict, C., Crandall, I., and Pillaid, D. R. (2012). Harmine is a Potent Antimalarial Targeting Hsp90 and Synergizes With Chloroquine and Artemisinin. Antimicrob. Agents Chemother. 56, 42074213. doi: 10.1128/AAC.00328-12

Tipton, K. A., Dimitrova, D., and Rather, P. N. (2015). Phase-Variable Control of Multiple Phenotypes in Acinetobacter Baumannii Strain AB5075. J. Bacteriol. 197, 2593-2599. doi: 10.1128/JB.00188-15

Valcek, A., Bogaerts, P., Denis, O., Huang, T.-D., and van der Henst, C. (2021). Molecular Epidemiology of Carbapenem-Resistant Acinetobacter Baumannii Strains in Belgian Acute-Care Hospitals. medRrxiv. doi: 10.1101/ 2021.07.06.21259728

Vallenet, D., Nordmann, P., Barbe, V., Poirel, L., Mangenot, S., Bataille, E., et al. (2008). Cruveiller S.Comparative Analysis of Acinetobacters: Three Genomes for Three Lifestyles. PloS One 3, e1805. doi: 10.1371/journal.pone.0001805

Weiner, L. M., Webb, A. K., Limbago, B., Dudeck, M. A., Patel, J., Kallen, A. J., et al. (2016). Antimicrobial-Resistant Pathogens Associated With HealthcareAssociated Infections: Summary of Data Reported to the National Healthcare Safety Network at the Centers for Disease Control and Prevention, 2011-2014. Infect. Control Hosp. Epidemiol. 37, 1288-1301. doi: 10.1017/ice.2016.174

Whiteway, C., Breine, A., Philippe, C., and van der Henst, C. (2021). Acinetobacter Baumannii. Trends Microbiol. doi: 10.1016/j.tim.2021.11.008

Wieland, K., Chhatwal, P., and Vonberg, R. P. (2018). Nosocomial Outbreaks Caused by Acinetobacter Baumannii and Pseudomonas Aeruginosa: Results of a Systematic Review. Am. J. Infect. Control 46, 643-648. doi: 10.1016/ j.ajic.2017.12.014

Wong, D., Nielsen, T. B., Bonomo, R. A., Pantapalangkoor, P., Luna, B., and Spellberg, B. (2017). Clinical and Pathophysiological Overview of Acinetobacter Infections: A Century of Challenges. Clin. Microbiol. Rev. 30, 409-447. doi: 10.1128/CMR.00058-16

World Health Organization (2017). Global Priority List of Antibiotic-Resistant Bacteria to Guide Research, Discovery, and Development of New Antibiotics Genève, Switzerland: WHO.

Wright, M. S., Iovleva, A., Jacobs, M. R., Bonomo, R. A., and Adams, M. D. (2016). Genome Dynamics of Multidrug-Resistant Acinetobacter Baumannii During Infection and Treatment. Genome Med. 8, 1-12. doi: 10.1186/s13073-0160279-y

Xia, Y., Qi, Y. M., Yu, X. H., Wang, B. F., Cao, R. H., and Jiang, D. X. (2019). Nematicidal Effect Against Bursaphelenchus Xylophilus of Harmine Quaternary Ammonium Derivatives, Inhibitory Activity and Molecular Docking Studies on Acetylcholinesterase. Eur. J. Plant Pathol. 153, 111-122. doi: 10.1007/s10658-018-1559-8

Zeidler, S., and Müller, V. (2019). Coping With Low Water Activities and Osmotic Stress in Acinetobacter Baumannii: Significance, Current Status and Perspectives. Environ. Microbiol. 21, 2212-2230. doi: 10.1111/1462-2920.14565 
Zhang, L., Li, D., and Yu, S. (2020). Pharmacological Effects of Harmine and its Derivatives: A Review. Arch. Pharm. Res. 43, 1259-1275. doi: 10.1007/s12272-020-01283-6

Conflict of Interest: The authors declare that the research was conducted in the absence of any commercial or financial relationships that could be construed as a potential conflict of interest.

Publisher's Note: All claims expressed in this article are solely those of the authors and do not necessarily represent those of their affiliated organizations, or those of the publisher, the editors and the reviewers. Any product that may be evaluated in this article, or claim that may be made by its manufacturer, is not guaranteed or endorsed by the publisher.

Copyright $\odot 2022$ Breine, Van Gysel, Elsocht, Whiteway, Philippe, Quinet, Valcek, Wouters, Ballet and Van der Henst. This is an open-access article distributed under the terms of the Creative Commons Attribution License (CC BY). The use, distribution or reproduction in other forums is permitted, provided the original author(s) and the copyright owner(s) are credited and that the original publication in this journal is cited, in accordance with accepted academic practice. No use, distribution or reproduction is permitted which does not comply with these terms. 